TRANSACTIONS OF THE

AMERICAN MATHEMATICAL SOCIETY

Volume 349, Number 10, October 1997, Pages 3989-4006

S $0002-9947(97) 01833-3$

\title{
MONGE-AMPÈRE EQUATIONS RELATIVE TO A RIEMANNIAN METRIC
}

\author{
A. ATALLAH AND C. ZUILY
}

\begin{abstract}
We prove that in a bounded strictly convex open set $\Omega$ in $\mathbb{R}^{n}$, the problem$$
\left\{\begin{array}{l}
\operatorname{det} \nabla^{2} u=f(x) \\
\left.u\right|_{\partial \Omega}=\varphi
\end{array}\right.
$$

where $f>0, f \in C^{\infty}(\bar{\Omega}), \varphi \in C^{\infty}(\partial \Omega)$, has a unique strictly convex solution $u \in C^{\infty}(\bar{\Omega})$. This result extends to an arbitrary metric a theorem which has been proved by Caffarelli-Nirenberg-Spruck in the case of the Euclidean metric.
\end{abstract}

\section{INTRODUCTION}

This work deals with the Dirichlet problem for Monge-Ampère equations relative to an arbitrary smooth Riemannian metric, and its goal is to provide an exact analogue to a result of Caffarelli-Nirenberg-Spruck [CNS] in the case of the flat metric. More precisely let $g$ be a $C^{\infty}$ Riemannian metric on a smooth bounded open set $\Omega$ in $\mathbb{R}^{n}, n \geq 2$. Let $\nabla u$ and $\nabla^{2} u$ denote the covariant derivative and the Hessian of $u$ relative to the Levi-Civita connexion of the metric $g$. We shall call the functions $u$ for which the Hessian $\left(\nabla^{2} u\right)$ is positive definite strictly convex.

We shall assume that $\Omega$ is strictly convex in the following sense:

(I.1) there exists $h \in C^{\infty}(\bar{\Omega})$ strictly convex in $\bar{\Omega}$ such that $h=0$ on $\partial \Omega$.

The main result of this paper is then

Theorem I.1. Let $\Omega$ be a $C^{\infty}$ strictly convex bounded open set in $\mathbb{R}^{n}, n \geq 2$.

Given $f \in C^{\infty}(\bar{\Omega}), f>0$ on $\bar{\Omega}$, and $\varphi \in C^{\infty}(\partial \Omega)$, the problem

$$
\left\{\begin{array}{l}
\operatorname{det} \nabla^{2} u(x)=f(x) \text { in } \Omega, \\
u_{\mid \partial \Omega}=\varphi
\end{array}\right.
$$

has a unique strictly convex solution $u \in C^{\infty}(\bar{\Omega})$.

The interest in such equations is related to the fact that a few problems in differential geometry (as Minkowski and Weyl problems; see also [GS]) lead to equations of this type. The case of the flat metric in $\mathbb{R}^{n}$ has been widely investigated during the past years, and Theorem I.1 in this case has been proved by CaffarelliNirenberg-Spruck [CNS].

In the two dimensionnal case, for general metrics, this result has been proved by Corona $[\mathrm{C}]$, who assumes moreover the existence of an upper solution, by Hong $[\mathrm{H}]$

Received by the editors March 6, 1995 and, in revised form, November 28, 1995.

1991 Mathematics Subject Classification. Primary 35J65, 35Q99.

(C)1997 American Mathematical Society 
in the case $\varphi=0$ and by Atallah [A] in the general case. Recently Guan-Spruck [GS] investigated related equations for metrics on the sphere when subsolutions exist. Finally we would like to mention the recent results by Atallah $[\mathrm{A}]$ in the case where $f=f(x, u, \nabla u)$, assuming the existence of upper and subsolutions.

The proof, as usual, uses the continuity method, which leads to uniform bounds for the $C^{2+\alpha}(\bar{\Omega})$ norms of smooth solutions of (I.2) (see [CNS] or [GT]). By results of Evans [E] and Krylov [KR] such bounds can be deduced from $C^{2}$ uniform bounds. The interior estimates and the estimates of the tangential and mixed second derivatives on the boundary, which rely on the previous work mentioned above, appear already in $[\mathrm{A}]$, and we give them here for the sake of completeness. The main part of the paper is then devoted to bounding the pure normal derivative on the boundary, which leads to the construction of a local upper solution for the problem (I.1). This construction, which is achieved under an appropriate set of coordinates, is inspired by [CNS] but requires here a careful and more technical proof.

\section{Proof of TheOREM I.1}

A constant $C$ will be called under control if it depends only on $n, \Omega, g, f, \varphi$. A function $u$ is said to be under control if it is bounded by such a constant. Moreover, we shall denote by $C$ a constant which may vary from line to line but is always under control.

\section{1. $C^{0}$ AND $C^{1}$ BOUNDS}

First of all, by (I.1), if $\lambda$ is a large enough positive constant, the function

$$
\psi=\lambda h+\varphi
$$

is a subsolution for problem (I.2). By the maximum principle we get

$$
\psi \leq u \leq \max _{\partial \Omega} \varphi \text { in } \bar{\Omega} \text {. }
$$

On the other hand it is easy to see that $|\nabla u|^{2}=\sum g^{i j} \nabla_{i} u \nabla_{j} u$ achieves its maximum on the boundary. Since tangential derivatives of $u$ on $\partial \Omega$ are obviously under control, the $C^{1}$ bounds in $\bar{\Omega}$ will follow from the control of the normal derivative $\frac{\partial u}{\partial \nu}$ on the boundary; here $\nu$ is the interior normal. By the Hopf maximum principle and (II.2) we get

$$
\frac{\partial \psi}{\partial \nu} \leq \frac{\partial u}{\partial \nu} \text { on } \partial \Omega
$$

For the upper bound we follow [C]. A geodesic $\gamma$ normal to $\partial \Omega$ at $p$ intersects $\partial \Omega$ at one other point $q$, by the strict convexity of $\Omega$. Let $w$ be the linear function on $\gamma$ equal to $\varphi$ at $p$ and $q$, and $\dot{w}$ its gradient, which is a continuous function. Since $u$ is strictly convex on $\gamma$, the maximum principle implies that $u \leq w$ on $\gamma \cap \Omega$ and $\frac{\partial u}{\partial \nu}(p) \leq \dot{w}(p) \leq \inf _{\partial \Omega} \dot{w}$.

\section{2. $C^{2}$ ESTimates ON THE BOUNDARY}

We shall prove pointwise bounds for the second derivatives on the boundary. Let $p$ be a point on $\partial \Omega$; it can be taken as the origin of coordinates. Using the function $h$ given by (I.1), the maximum principle and a system of normal coordinates near 
the origin, one see easily that in a neighborhood $V$ of the origin, the boundary is given by

$$
\partial \Omega \cap V=\left\{\left(x_{1}, \ldots, x_{n}\right): x_{n}=\rho\left(x_{1}, \ldots, x_{n-1}\right)\right\},
$$

where $\rho$ is a smooth function such that

$$
\rho\left(x^{\prime}\right)=\frac{1}{2} \sum_{i=1}^{n-1} k_{i} x_{i}^{2}+O\left(\left|x^{\prime}\right|^{3}\right), \quad k_{i}>0, \quad x^{\prime}=\left(x_{1}, \ldots, x_{n-1}\right) .
$$

Estimates for the pure tangential derivatives. The vector field

$$
X_{\alpha}=\frac{\partial}{\partial x_{\alpha}}+\frac{\partial \rho}{\partial x_{\alpha}} \frac{\partial}{\partial x_{n}}
$$

is tangent to the boundary if $\alpha=1,2, \ldots, n-1$, and

$$
\nabla_{\alpha \beta} u(0)=\left(X_{\alpha} X_{\beta} \varphi\right)(0)-\frac{\partial^{2} \rho}{\partial x_{\alpha} \partial x_{\beta}}(0) \frac{\partial u}{\partial x_{n}}(0) .
$$

This shows, using the $C^{1}$ bounds, that $\nabla_{\alpha \beta} u(0)$ is under control.

Estimates for the mixed second derivatives. Differentiating the identity $\log \operatorname{det} \nabla_{i j} u=\log f=\Phi$, one gets

$$
u^{i j} \nabla_{i j k} u=\nabla_{k} \Phi, \quad k=1, \ldots, n,
$$

where $\left(u^{i j}\right)=\left(\nabla_{i j} u\right)^{-1}$ and the summation convention has been used. Let us set $L=u^{i j} \nabla_{i j}$ and let $X=\sum_{k=1}^{n} b_{k} \nabla_{k}$ be a smooth vector field. Then

$$
L(X u)=u^{i j}\left(\nabla_{i j} b_{k}\right) \nabla_{k} u+2 u^{i j} \nabla_{i} b_{k} \nabla_{k j} u+u^{i j} b_{k} \nabla_{i j k} u+u^{i j} b_{k}\left(\nabla_{k i j} u-\nabla_{i j k} u\right) .
$$

By the well known Ricci formulas and (II.6) one gets

$$
L(X u)=u^{i j} \underbrace{\left(\nabla_{i j} b_{k}\right) \nabla_{k} u}_{(1)}+\underbrace{2 u^{i j} \nabla_{i} b_{k} \nabla_{k j} u}_{(2)}+\underbrace{b_{k} \nabla_{k} \Phi}_{(3)}+\underbrace{u^{i j} b_{k} R_{j k i}^{s} \nabla_{s} u}_{(4)}
$$

where $R_{j k i}^{s}$ are the components of the Riemann curvature tensor, which depends only on the metric $g$. By the $C^{1}$ estimates we can write

$$
\text { (1) }+(4)=u^{i j} A_{i j}
$$

where the $A_{i j}$ are under control.

Now, since $u^{i j} \nabla_{k j} u=\delta_{i k}$, we get

$$
(2)=2 \nabla_{i} b_{i}
$$

It follows that $L X u=A_{0}+A_{i j} u^{i j}$, where $A_{0}$ and $A_{i j}$ are under control; therefore $|L(X(u-\varphi))| \leq C_{1}+C_{2} \sum_{i=1}^{n} u^{i i}$ since $\left(u^{i j}\right)>0$. Now

$$
\sum_{i=1}^{n} u^{i i} \geq n\left(\operatorname{det} u^{i j}\right)^{1 / n}=n f^{-1 / n} \geq C_{3}>0
$$

which implies $|L(X(u-\varphi))| \leq C_{4} \sum_{i=1}^{n} u^{i i}$. 
Now by the strict convexity of the function $h$ given by (I.1) we get $u^{i j} \nabla_{i j} h \geq$ $C_{5} \sum_{i=1}^{n} u^{i i}$. It follows that, if $\lambda$ is large enough,

$$
\lambda L h-|L(X(u-\varphi))|>0 \text { in } \bar{\Omega} .
$$

Let us assume now that $X$ is tangent to the boundary; then $\left.X(u-\varphi)\right|_{\partial \Omega}=\left.h\right|_{\partial \Omega}=0$. It follows from the Hopf maximum principle that

$$
\left|\frac{\partial}{\partial \nu}(X(u-\varphi))\right| \leq-\lambda \frac{\partial h}{\partial \nu} \text { on } \partial \Omega .
$$

Taking $X=\partial_{\alpha}+\rho_{\alpha} \partial_{n}$, we get

$$
\left|\nabla_{n \alpha} u(0)\right| \leq C, \quad \alpha=1, \ldots, n-1 .
$$

Estimates for the pure normal derivative. As before, let $p$ be a point on $\partial \Omega$ that we take as the origin. We shall work in a special set of coordinates in a neighborhood $V$ of the origin. Let $\nu(p)$ be the interior unit normal (with respect to $g$ ) to $\partial \Omega$ at $p \in V \cap \partial \Omega$. Let us consider first the case $n \geq 3$. Let $X_{1}, \ldots, X_{n-1}$ be a basis of the tangent space to $\partial \Omega$ at the origin. A point $p \in \partial \Omega \cap V$ will be described by its geodesic coordinates, i.e. $p=\exp _{0} \sum_{i=1}^{n-1} x_{i} X_{i}$. Now let us consider the map $H: \mathbb{R}^{n-1} \times \mathbb{R}_{+} \rightarrow V$,

$$
H\left(x_{1}, \ldots, x_{n-1}, x_{n}\right)=\exp _{p} x_{n} \nu(p), \quad p=\exp _{0} \sum_{i=1}^{n-1} x_{i} X_{i} .
$$

This map is a diffeomorphism from a neighborhood $W_{0} \times[0, \varepsilon]$ of the origin in $\mathbb{R}^{n-1} \times \mathbb{R}_{+}$to a neighborhood $V$ of the origin in $\bar{\Omega}$. In this system of coordinates $\partial \Omega \cap V=\left\{\left(x_{1}, \ldots, x_{n}\right): x_{n}=0\right\}$. If $n=2$ let the boundary be given by a curve $\alpha(t)$ parametrized by the arc length; then we consider $H\left(t, x_{2}\right)=\exp _{\alpha(t)} x_{2} \nu(t)$. In these coordinates the metric $g$ and the Christoffel symbols satisfy the set of relations

$$
\begin{cases}\text { i) } & g=d x_{n}^{2}+\sum_{i, j=1}^{n-1} g_{i j} d x_{i} d x_{j} \\ \text { ii) } & \Gamma_{i j}^{k}(0)=0, \quad 1 \leq i, j, k \leq n-1 \\ \text { iii) } & \Gamma_{i j}^{n}(0)=k_{i} \delta_{i j}, \quad 1 \leq i, j \leq n-1, \text { where } k_{i} \text { are the principal } \\ & \text { curvatures of the boundary at the origin; } k_{i}>0 \text { by }(\text { I. } 1) \\ \text { iv) } & \Gamma_{i n}^{k}(0)=-k_{i} \delta_{i k}, \quad 1 \leq i, k \leq n-1 \\ \text { v) } & \Gamma_{i n}^{n}(0)=0, \quad i=1, \ldots, n-1 \\ \text { vi) } & \Gamma_{n n}^{k}(x)=0, \quad 1 \leq k \leq n, \quad x \in V .\end{cases}
$$

In these coordinates

$$
\left.\frac{\partial^{2} u}{\partial \nu^{2}}(0)=\nabla_{n n} u(0)=\frac{\partial^{2} u}{\partial x_{n}^{2}}(0) \quad \text { by vi }\right)
$$

Now our equation (I.2), which is invariant, can be written

$$
\nabla_{n n} u(0) \cdot \operatorname{det}\left(\left(\nabla_{i j} u(0)\right)_{1 \leq i, j \leq n-1}\right)+G\left(\left(\nabla_{i j} u(0)\right)_{(i, j) \neq(n, n)}\right)=f .
$$


By the preceding estimates, $G$ is under control. Therefore a uniform upper bound for $\nabla_{n n} u(0)$ will follow from a uniform lower bound for $\operatorname{det}\left(\nabla_{i j} u(0)\right)_{1 \leq i, j \leq n-1}$. We shall show that

$$
\sum_{i, j=1}^{n-1} \nabla_{i j} u(0) \xi_{i} \xi_{j} \geq C_{0}|\xi|^{2},
$$

where $C_{0}>0$ depends only on $\Omega, n, g, \varphi, f$. This will follow from $\nabla_{i i} u(0) \geq C_{1}>0$, $i=1, \ldots, n-1$. By symetry it is therefore enough to show that $\nabla_{11} u(0)$ is uniformly bounded below. Now by (II.9) iii),

$$
\nabla_{11} u(0)=u_{11}(0)-k_{1} u_{n}(0)=\varphi_{11}(0)-k_{1} u_{n}(0) .
$$

Since we do not have any control of the lower bound of $\varphi_{11}(0)$ we are going to replace $u$ by $v=u+w$, where $w$ will be chosen such that $\nabla_{11} v(0)=\nabla_{11} u(0)=-k_{1} v_{n}(0)$, and we shall work with $v$.

Lemma II.1. One can find, in a neighborhood of the origin, a smooth convex function $w$ such that $w(0)=-\varphi(0)$ and

i) $\nabla_{11} w(0)=0$,

ii) $w_{i}(0)=-\varphi_{i}(0), i=1, \ldots, n-1$,

iii) $w_{11}(0)=-\varphi_{11}(0)$,

iv) $\nabla_{i j} w(0)=\delta_{i j}, 2 \leq i, j \leq n$,

v) $\nabla_{1 j} w(0)=0,2 \leq j \leq n$.

Proof. Recall that

$$
\nabla_{11}=\frac{\partial^{2}}{\partial x_{1}^{2}}-\sum_{i=1}^{n} \Gamma_{11}^{i} \frac{\partial}{\partial x_{i}}
$$

Let $a_{i}$ be the quadratic part of the Taylor expansion of $\Gamma_{11}^{i}$ at the origin, i.e., for small $|x|$,

$$
\Gamma_{11}^{i}(x)=a_{i}(x)+O\left(|x|^{3}\right), \quad i=1, \ldots, n .
$$

Then by (II.9) iii), $a_{n}(0)=k_{1}, a_{k}(0)=0, k=1, \ldots, n-1$.

Let us solve the analytic Cauchy problem

$$
\left\{\begin{aligned}
\frac{\partial^{2} w}{\partial x_{1}^{2}}-\sum_{k=1}^{n} & a_{k}(x) \frac{\partial w}{\partial x_{k}}=\lambda|x|^{2} \\
\left.w\right|_{x_{1}=0}= & -\varphi(0)-\sum_{i=2}^{n-1} \varphi_{i}(0) x_{i}-\frac{1}{k_{1}} \varphi_{11}(0) x_{n} \\
& +\frac{1}{2} \sum_{i=2}^{n-1}\left(1-\frac{k_{i}}{k_{1}} \varphi_{11}(0)\right) x_{i}^{2} \\
& +\sum_{i=2}^{n-1} k_{i} \varphi_{i}(0) x_{i} x_{n}+\frac{1}{2} x_{n}^{2} \\
\left.\frac{\partial w}{\partial x_{1}}\right|_{x_{1}=0} & -\varphi_{1}(0)+k_{1} \varphi_{1}(0) x_{n}
\end{aligned}\right.
$$

Then for any $\lambda$ the conditions i) to $\mathrm{v}$ ) are satisfied. We just have to show that $w$ is convex, i.e., $\left(\nabla_{i j} w\right) \geq 0$ if $\lambda$ is large enough. We have $\nabla_{11} w=\lambda|x|^{2}+O\left(|x|^{3}\right)$. $O(|\nabla w|)$. Now for $(i, j) \neq(1,1) \nabla_{i j} w-\delta_{i j}$ vanishes at the origin; its linear part 
is $\sum_{k=1}^{n} \frac{\partial}{\partial x_{k}}\left(\nabla_{i j} w\right)(0) x_{k}$. Since $\lambda$ occurs in the equation with a quadratic term $|x|^{2}$ and the data are independant of $\lambda$, this linear part is independant of $\lambda$. Therefore

$$
\left|\nabla_{i j} w-\delta_{i j}\right| \leq C_{1}|x|+C_{2}(\lambda)|x|^{2}, \quad(i, j) \neq(1,1),
$$

where $C_{1}$ depends only on the data in (II.11). Taking $\lambda$ large enough and then $|x|$ small, we get easily

$$
\sum_{i, j=1}^{n} \nabla_{i j} w(x) \xi_{i} \xi_{j} \geq \frac{\lambda}{2}|x|^{2} \xi_{1}^{2}+\frac{1}{2} \sum_{i=2}^{n} \xi_{i}^{2} \geq 0
$$

Recall that we had to prove that $\nabla_{11} u(0) \geq C_{0}>0$. Now by Lemma II.1

$$
\nabla_{11} u(0)=\nabla_{11} v(0)=\left(v_{11}(0)-\sum_{k=1}^{n} \Gamma_{11}^{k}(0) v_{k}(0)\right)=v_{11}(0)-k_{1} v_{n}(0)=-k_{1} v_{n}(0)
$$

since $v_{11}(0)=\varphi_{11}(0)+w_{11}(0)=0$ by iii).

Therefore we are lead to prove the uniform bound

$$
v_{n}(0) \leq-\varepsilon_{0}<0 .
$$

This will be proved by constructing an appropriate local upper solution.

Lemma II.2. For small $\delta>0$ one can find $\varepsilon_{0}>0$ and a smooth function $\rho$ in $V=\left\{\left(x_{1}, \ldots, x_{n}\right): 0 \leq x_{n} \leq \delta, 0 \leq x_{n}+\sum_{i=1}^{n-1} \frac{k_{i}}{4} x_{i}^{2} \leq \delta\right\}$ such that:

i) $\rho(0)=0, \frac{\partial \rho}{\partial x_{n}}(0)=-\varepsilon_{0}$,

ii) $\left\{\begin{array}{l}\operatorname{det} \nabla_{i j} \rho<\operatorname{det} \nabla_{i j} v \text { in } V, \\ v \leq \rho \text { on } \partial V\end{array}\right.$

Let us first show how Lemma II.2 implies (II.12). We use this weak form of the maximum principle.

Lemma II.3. In a bounded open set $V$ let there be given a smooth convex function $v$ (i.e. $\left(\nabla_{i j} v\right) \geq 0$ ) and a smooth function $\rho$ such that

$$
\left\{\begin{array}{l}
\operatorname{det} \nabla_{i j} v(x)>\operatorname{det} \nabla_{i j} \rho(x) \text { in } V \\
v \leq \rho \text { on } \partial V
\end{array}\right.
$$

Then $v \leq \rho$ in $V$.

Proof. Note that $\rho$ is not assumed to be convex. We prove this lemma by contradiction. Suppose there is $\bar{x}$ in $V$ such that $\rho(\bar{x})<v(\bar{x})$. The continuous function $\rho-v$, which is nonnegative on $\partial \Omega$ and $<0$ at $\bar{x}$, will achieve its absolute minimum at $x_{0} \in V$. It follows that $\left(\left(\nabla_{i j} \rho-\nabla_{i j} v\right)\left(x_{0}\right)\right) \geq 0$, so $\left(\nabla_{i j} \rho\right)\left(x_{0}\right) \geq\left(\nabla_{i j} v\right)\left(x_{0}\right)$. But this implies $\operatorname{det}\left(\nabla_{i j} \rho\left(x_{0}\right)\right) \geq \operatorname{det}\left(\nabla_{i j} v\left(x_{0}\right)\right)$, which is a contradiction.

Assuming Lemma II.2, it follows from Lemma II.3 that $v\left(0, x_{n}\right) \leq \rho\left(0, x_{n}\right)$ for $x_{n}>0$, and since $v(0,0)=\rho(0,0)=0$ we deduce that $\frac{\partial v}{\partial x_{n}}(0) \leq \frac{\partial \rho}{\partial x_{n}}(0)=-\varepsilon_{0}$, and (II.12) is proved. 
Proof of Lemma II.2. We can write, with $x^{\prime}=\left(x_{2}, \ldots, x_{n-1}\right)$,

$$
\begin{aligned}
v\left(x^{\prime}, 0\right)= & \varphi\left(x^{\prime}\right)+w\left(x^{\prime}, 0\right) \\
= & \varphi(0)+w(0)+\sum_{i=1}^{n-1}\left(\varphi_{i}(0)+w_{i}(0)\right) x_{i}+\frac{1}{2}\left(\varphi_{11}(0)+w_{11}(0)\right) x_{1}^{2} \\
& +\sum_{j=2}^{n-1} a_{j} x_{1} x_{j}+O\left(\sum_{j=2}^{n-1} x_{j}^{2}\right)+\frac{b k_{1}}{3} x_{1}^{3}+O\left(\sum_{j=1}^{n-1} x_{j}^{4}\right) .
\end{aligned}
$$

It follows from Lemma II.1 that

$$
v\left(x^{\prime}, 0\right)=\sum_{j=2}^{n-1} a_{j} x_{1} x_{j}+\frac{1}{3} b k_{1} x_{1}^{3}+O\left(\sum_{j=2}^{n-1} x_{j}^{2}\right)+O\left(\sum_{j=1}^{n-1} x_{j}^{4}\right),
$$

where $a_{j}$ and $b$ depend only on the data and the $O$ are under control. We shall set, with $\varepsilon_{0}=\delta^{3 / 2}$,

$$
\begin{aligned}
\rho\left(x^{\prime}, x_{n}\right)= & -\varepsilon_{0} x_{n}+\sum_{j=2}^{n-1} a_{j} x_{1} x_{j}+B \sum_{j=2}^{n-1} x_{j}^{2} \\
& +\frac{\delta}{2 K}\left(b x_{1}+\frac{K}{\delta}\left(x_{n}+\sum_{i=1}^{n-1} \frac{k_{i}}{4} x_{i}^{2}\right)\right)^{2}+\frac{b k_{1}}{12} x_{1}^{3},
\end{aligned}
$$

where the small letters $b, a_{j}$ are fixed by (II.13) and the big $B, K$ are to be chosen.

First claim. $v \leq \rho$ on $\partial V$.

- If $x_{n}=0$ and $\sum_{i=1}^{n-1} \frac{k_{i}}{4} x_{i}^{2} \leq \delta$, using (II.13) and (II.14) we get

$$
\rho-v \geq(B-C) \sum_{j=2}^{n-1} x_{j}^{2}+\frac{K}{2 \delta}\left(\sum_{j=1}^{n-1} \frac{k_{j}}{4} x_{j}^{2}\right)^{2}-C \sum_{j=1}^{n-1} x_{j}^{4},
$$

since for $j=2, \ldots, n-1$

$$
\left|x_{1} x_{j}^{2}\right| \leq \frac{1}{2}\left(\sum_{j=2}^{n-1} x_{j}^{2}+\sum_{j=1}^{n-1} x_{j}^{4}\right) .
$$

Taking $B \geq 2 C, \delta<1$ and $\frac{k_{j}^{2}}{16} K \geq 3 C$, we get $\rho-v \geq 0$ on this part of $\partial V$.

- $\quad$ On $x_{n}+\sum_{i=1}^{n-1} \frac{k_{i}}{4} x_{i}^{2}=\delta$, since $v=u+w$ and we have uniform bounds for the first derivatives of $u$ on $\bar{\Omega}$, we can write

$$
v\left(x^{\prime}, x_{n}\right)=v\left(x^{\prime}, 0\right)+x_{n} \tilde{v}, \quad 0 \leq x_{n} \leq \delta,
$$

where $\tilde{v}$ is under control. Therefore

$$
\begin{aligned}
\rho-v \geq & -\varepsilon_{0} x_{n}+B \sum_{j=2}^{n-1} x_{j}^{2}+\frac{\delta}{2 K}\left(b x_{1}+K\right)^{2} \\
& +\frac{b k_{1}}{12} x_{1}^{3}-\frac{b k_{1}}{3} x_{1}^{3}-C \sum_{j=2}^{n-1} x_{j}^{2}-C \sum_{j=1}^{n-1} x_{j}^{4}-C \delta .
\end{aligned}
$$


Now since $\left|x_{1}\right|=O\left(\delta^{1 / 2}\right)$ we can take $\delta$ so small that $\left|b x_{1}\right| \leq 1$. Taking $K \geq 2$ we get $\left(K+b x_{1}\right)^{2} \frac{\delta}{2 K} \geq \frac{K}{8} \delta$. Now we take $B \geq 2 C, \varepsilon_{0}<1$. Since in $V\left|x_{1}\right|^{3}+\sum_{j=1}^{n-1} x_{j}^{4} \leq C \delta$, one can find a constant under control such that $\rho-v \geq \frac{K}{8} \delta-C \delta$. This is positive if $K \geq 8 C$.

Second claim. We have, for $\delta$ small enough and $B$ large,

$$
\operatorname{det} \nabla_{i j} \rho \leq C \delta^{1 / 2} \text { in } V .
$$

Let us set $\lambda=\frac{\delta}{K}$ (which is small) and $\theta=\lambda \rho$. Then

$$
\begin{aligned}
\theta= & -\varepsilon_{0} \lambda x_{n}+\lambda \sum_{j=2}^{n-1} a_{j} x_{1} x_{j}+\lambda B \sum_{j=2}^{n-1} x_{j}^{2} \\
& +\frac{1}{2}\left(\lambda b x_{1}+x_{n}+\sum_{j=1}^{n-1} \frac{k_{j}}{4} x_{j}^{2}\right)^{2}+\frac{1}{12} \lambda b k_{1} x_{1}^{3} .
\end{aligned}
$$

Then

$$
\text { (II.15) } \Longleftrightarrow \operatorname{det} \nabla_{i j} \theta \leq C \lambda^{n} \delta^{1 / 2} .
$$

Let us introduce some notation. We shall denote by a dot the derivative with respect to $\lambda$. If $I=\left(i_{1} j_{1}, i_{2} j_{2}, \ldots, i_{k} j_{k}\right)$ is a multi-index of length $|I|=k$, we set

$$
\nabla_{I} \theta=\nabla_{i_{1} j_{1}} \theta \ldots \nabla_{i_{k} j_{k}} \theta
$$

therefore

$$
\nabla_{I} \dot{\theta}=\nabla_{i_{1} j_{1}} \dot{\theta} \ldots \nabla_{i_{k} j_{k}} \dot{\theta}
$$

We denote by $F$ the determinant function

$$
F\left(u_{i j}\right)=\operatorname{det} u_{i j}
$$

and set as usual

$$
\frac{\partial^{|I|} F}{\partial u_{I}}=\frac{\partial}{\partial u_{i_{1} j_{1}}} \ldots \frac{\partial F}{\partial u_{i_{k} j_{k}}}
$$

Let us mention an important thing. Since $F$ is the determinant function, $\frac{\partial F}{\partial u_{i j}}$ is independent of $u_{i \ell}$ and $u_{\ell j}$ for $\ell=1, \ldots, n$. Therefore $\frac{\partial^{|I|} F}{\partial u_{I}}$ vanishes unless $i_{1} \neq i_{2} \neq \ldots \neq i_{k}, j_{1} \neq j_{2} \neq \ldots \neq j_{k}$. Now by the Taylor formula we have

$$
\operatorname{det} \nabla_{i j} \theta=\sum_{k=0}^{n} \frac{1}{k !}\left(\frac{d}{d \lambda}\right)^{k}\left[F\left(\nabla_{i j} \theta(\lambda)\right)\right]_{\lambda=0} \lambda^{k}+\lambda^{n+1} G(x, \lambda) ;
$$

after that $\delta, B$ will be chosen such that the first term of the right hand side of (II.17) will be $O\left(\lambda^{n} \delta^{1 / 2}\right)$, and we shall have $G(x, \lambda)=O(1)$. Therefore

$$
G(x, \lambda) \lambda^{n+1}=\frac{\delta}{K} G(x, \lambda) \lambda^{n}=O\left(\lambda^{n} \delta^{1 / 2}\right) .
$$




\section{Lemma II.4.}

$$
\begin{aligned}
\left(\frac{d}{d \lambda}\right)^{k}\left[F\left(\nabla_{i j} \theta(\lambda)\right)\right]_{\lambda=0} & =\sum_{|I|=k} \frac{\partial^{k} F}{\partial u_{I}}\left(\nabla_{i j} \theta(0)\right) \nabla_{I} \dot{\theta}(0) \\
+ & \frac{k(k-1)}{2} \nabla_{11} \ddot{\theta}(0) \sum_{|I|=k-2} \frac{\partial^{k-1} F}{\partial u_{I} \partial u_{11}}\left(\nabla_{i j} \theta(0)\right) \nabla_{I} \dot{\theta}(0) \\
& +\sum_{\substack{q=q_{1}+q_{2} \\
q_{1}+2 q_{2}=k \\
q_{2} \geq 1}} \frac{k !}{q_{1} ! q_{2} !} \sum_{\substack{\left|I_{j}\right|=q_{j} \\
I_{2} \neq(11)}} \frac{\partial^{q} F}{\partial u_{I_{1}} \partial u_{I_{2}}}\left(\nabla_{i j} \theta(0)\right) \nabla_{I_{1}} \dot{\theta}(0) \frac{\nabla_{I_{2}} \ddot{\theta}(0)}{2^{q_{2}}}
\end{aligned}
$$

Proof. Let us recall the Faa di Bruno formula. If we set $(1)=\left(\frac{d}{d \lambda}\right)^{k}\left[F\left(\nabla_{i j} \theta(\lambda)\right)\right]$, then

$$
(1)=\sum \frac{k !}{q_{1} ! \ldots q_{k} !} \sum_{\left|I_{j}\right|=q_{j}} \frac{\partial^{q} F}{\partial u_{I_{1}} \ldots \partial u_{I_{k}}}\left(\nabla_{i j} \theta\right) \frac{\nabla_{I_{1}} \dot{\theta}}{1 ! q_{1}} \frac{\nabla_{I_{2}} \ddot{\theta}}{2 ! q_{2}} \ldots \frac{\nabla_{I_{k}} \ddot{\theta}(k)}{k ! q_{k}}
$$

where $q=q_{1}+\ldots+q_{k}$ and the first sum is taken for $q_{1}+2 q_{2}+\ldots+k q_{k}=k$. But $\left(\frac{d}{d \lambda}\right)^{\ell} \theta \equiv 0$ for $\ell \geq 3$, and therefore

$$
(1)=\sum_{\substack{q=q_{1}+q_{2} \\ q_{1}+2 q_{2}=k}} \frac{k !}{q_{1} ! q_{2} !} \sum_{\left|I_{j}\right|=q_{j}} \frac{\partial^{q} F}{\partial u_{I_{1}} \partial u_{I_{2}}}\left(\nabla_{i j} \theta\right) \nabla_{I_{1}} \dot{\theta} \cdot \frac{\nabla_{I_{2}} \ddot{\theta}}{2^{q_{2}}} .
$$

If $\left|I_{2}\right|=q_{2}=0$, the corresponding term in (1) is $\sum_{|I|=k} \frac{\partial^{k} F}{\partial u_{I}}\left(\nabla_{i j} \theta\right) \nabla_{I} \dot{\theta}$. If $\left|I_{2}\right|=q_{2}=$ 1 and $I_{2}=(11)$, then $q_{1}=k-2$ and the corresponding term in (1) is

$$
\sum_{|I|=k-2} \frac{k !}{(k-2) !} \frac{\partial^{k-1} F}{\partial u_{I} \partial u_{11}}\left(\nabla_{i j} \theta\right) \nabla_{I} \dot{\theta} \frac{\nabla_{11} \ddot{\theta}}{2} .
$$

The proof is complete.

It follows from (II.16) that

$$
\left\{\begin{array}{l}
\theta(0)=\frac{1}{2}\left(x_{n}+\sum_{j=1}^{n-1} \frac{k_{j}}{4} x_{j}^{2}\right)^{2}, \\
\dot{\theta}(0)=-\varepsilon_{0} x_{n}+\sum_{j=2}^{n-1} a_{j} x_{1} x_{j}+B \sum_{j=2}^{n-1} x_{j}^{2}+b x_{1}\left(x_{n}+\sum_{j=1}^{n-1} \frac{k_{j}}{4} x_{j}^{2}\right)+\frac{1}{12} b k_{1} x_{1}^{3}, \\
\ddot{\theta}(0)=b^{2} x_{1}^{2} .
\end{array}\right.
$$

Moreover using (II.9) we get in $V$

$$
\left\{\begin{array}{l}
\Gamma_{i j}^{k}=O\left(\delta^{1 / 2}\right), \quad 1 \leq i, j, k \leq n-1 \\
\Gamma_{i j}^{n}=k_{i} \delta_{i j}+O\left(\delta^{1 / 2}\right), \quad 1 \leq i, j \leq n-1 \\
\Gamma_{i n}^{k}=O(1), \quad 1 \leq i, k \leq n-1 \\
\Gamma_{i n}^{n}=O\left(\delta^{1 / 2}\right), \quad 1 \leq i \leq n-1 \\
\Gamma_{n n}^{k} \equiv 0, \quad 1 \leq k \leq n
\end{array}\right.
$$

Finally we recall that $\nabla_{i j}=\frac{\partial^{2}}{\partial x_{i} \partial x_{j}}-\sum_{k=1}^{n} \Gamma_{i j}^{k} \frac{\partial}{\partial x_{k}}$. 
Now it is easy to see that

$$
\left\{\begin{array}{l}
\nabla_{i j} \theta(0)=\frac{k_{i} k_{j}}{4} x_{i} x_{j}-\frac{k_{i}}{2} \delta_{i j} \Gamma+\sigma_{i j}, \quad 1 \leq i, j \leq n-1, \\
\nabla_{i n} \theta(0)=\frac{k_{i}}{2} x_{i}+\sigma_{i n}, \quad 1 \leq i \leq n-1, \\
\nabla_{n n} \theta(0)=1
\end{array}\right.
$$

where

$$
\left\{\begin{array}{l}
\Gamma=x_{n}+\sum_{j=1}^{n-1} \frac{k_{j}}{4} x_{j}^{2} \\
\sigma_{i j}=O\left(\Gamma^{3 / 2}\right)
\end{array}\right.
$$

It is easy to simplify $\operatorname{det} \nabla_{i j} \theta(0)$. Let us set

$$
\left\{\begin{array}{l}
c_{i j}=\nabla_{i j} \theta(0)-\left(\frac{k_{i}}{2} x_{i}+\sigma_{i n}\right) \nabla_{n j} \theta(0), \quad 1 \leq i \leq n-1, \quad 1 \leq j \leq n \\
c_{n j}=\nabla_{n j} \theta(0), \quad 1 \leq j \leq n
\end{array}\right.
$$

then

$$
\operatorname{det} \nabla_{i j} \theta(0)=\operatorname{det} c_{i j}
$$

Now we set

$$
\left\{\begin{array}{l}
d_{i j}=c_{i j}-\left(\frac{k_{j}}{2} x_{j}+\sigma_{n j}\right) c_{i n}, \quad 1 \leq i \leq n, \quad 1 \leq j \leq n-1 \\
d_{i n}=c_{i n}, \quad 1 \leq i \leq n
\end{array}\right.
$$

then

$$
\operatorname{det} d_{i j}=\operatorname{det} c_{i j}=\operatorname{det} \nabla_{i j} \theta(0) .
$$

Using the chain rule and an induction over $|I|$, we easily prove

\section{Lemma II.5.}

$$
\frac{\partial^{|I|} F}{\partial u_{I}}\left(\nabla_{i j} \theta(0)\right)=\frac{\partial^{|I|} F}{\partial u_{I}}\left(d_{i j}\right)+\sum_{|J|=|I|} c_{I J}(x) \frac{\partial^{|J|} F}{\partial u_{J}}\left(d_{i j}\right),
$$

where $c_{I J}$ are smooth functions satisfying $c_{I J}=O\left(\Gamma^{1 / 2}\right)$.

Using (II.20), (II.21), (II.22) and (II.24) we see easily that

$$
\left\{\begin{array}{l}
d_{i j}=-\frac{k_{i}}{2} \delta_{i j} \Gamma+\sigma_{i j}, \quad 1 \leq i, j \leq n-1, \\
d_{i n}=d_{n j}=0, \quad 1 \leq i, j \leq n-1 \\
d_{n n}=1
\end{array}\right.
$$

Now we have

Lemma II.6. For $|I| \leq n-2$ one has

$$
\frac{\partial^{|I|} F}{\partial u_{I}}\left(\nabla_{i j} \theta(0)\right)=c_{I} \Gamma^{n-|I|-1}+O\left(\Gamma^{n-|I|-1+1 / 2}\right),
$$

where $c_{I}$ is a real constant.

Moreover, if $|I|=n-2$ we have $c_{I}<0$. 
Proof. By Lemma II.5 the above formula will be implied by the same formula for $\frac{\partial^{|I|} F}{\partial u_{I}}\left(d_{i j}\right)$. Let us multiply the last line of the matrix $\left(d_{i j}\right)$ by $\Gamma$. We get a matrix $\left(\tilde{d}_{i j}\right)$, where $\tilde{d}_{i j}=d_{i j}$ if $(i, j) \neq(n, n)$ and $\tilde{d}_{n n}=\Gamma$. Now $F\left(\tilde{d}_{i j}\right)$ is homogeneous of order $n$ in the $\tilde{d}_{i j}$ 's; therefore $\frac{\partial^{|I|} F}{\partial u_{I}}\left(\tilde{d}_{i j}\right)=(1)$ is homogeneous of order $n-|I|$. A term of (1) is either made of diagonal term or it has at least one nondiagonal term. In the first case it is equal to cte $\Gamma^{n-|I|}+O\left(\Gamma^{n-|I|+1 / 2}\right)$; in the second case it is bounded by cte $\Gamma^{n-|I|-1} \Gamma^{3 / 2}=O\left(\Gamma^{n-|I|+1 / 2}\right)$. Dividing by $\Gamma$, we get the result. If $|I|=n-2, \frac{\partial^{|I|} F}{\partial u_{I}}\left(d_{i j}\right)$ is homogeneous of order two; its principal term is of the form $d_{i i} d_{j j}$; if $1 \leq i, j \leq n-1$ this term is $O\left(\Gamma^{2}\right)$, so the main term is $d_{i i} d_{n n}=-\frac{k_{i}}{2} \Gamma$. $\diamond$

Next, using (II.18), we get

$$
\left\{\begin{array}{l}
\nabla_{11} \dot{\theta}(0)=b k_{1} x_{1}+\gamma_{11}, \\
\nabla_{1 j} \dot{\theta}(0)=a_{j}+\frac{1}{2} b k_{j} x_{j}+\gamma_{1 j}, \quad 1 \leq j \leq n-1, \\
\nabla_{1 n} \dot{\theta}(0)=b+B O\left(\Gamma^{1 / 2}\right), \\
\nabla_{i n} \dot{\theta}(0)=B O\left(\Gamma^{1 / 2}\right), \quad 2 \leq i \leq n-1, \\
\nabla_{i j} \dot{\theta}(0)=\left[2 B-\frac{1}{2} b k_{j} x_{1}\right] \delta_{i j}+\gamma_{i j}, \quad 2 \leq i, j \leq n-1, \\
\nabla_{n n} \dot{\theta}(0)=0
\end{array}\right.
$$

where

$$
\gamma_{i j}=B O(\Gamma)+O\left(\delta^{3 / 2}\right) .
$$

Now we use (II.17), Lemmas II.4 and II.6 and (II.27) to compute $\operatorname{det} \nabla_{i j} \theta(\lambda)$. The integer $k$ will refer to (II.17). We set

$$
\begin{aligned}
A_{k}=\lambda^{k}[ & \sum_{|I|=k} \frac{\partial^{k} F}{\partial u_{I}}\left(\nabla_{i j} \theta(0)\right) \nabla_{I} \dot{\theta}(0) \\
& \left.+k(k-1)\left[b^{2}+O\left(\Gamma^{1 / 2}\right)\right] \sum_{|I|=k-2} \frac{\partial^{k-1} F}{\partial u_{I} \partial u_{11}}\left(\nabla_{i j} \theta(0)\right) \nabla_{I} \dot{\theta}(0)\right]
\end{aligned}
$$

since by (II.18) $\nabla_{11} \ddot{\theta}(0)=2 b^{2}+O\left(\Gamma^{1 / 2}\right)$.

Case 1. $k \leq n-3$.

Taking $\Gamma \leq 1$ and $B \geq 1$, we get from (II.27) $\left|\nabla_{i j} \dot{\theta}(0)\right| \leq c_{0} B$. Then Lemma II.6 implies

$$
\left|A_{k}\right| \leq C_{k} \lambda^{k} B^{k} \Gamma^{n-k-1}, \quad 0 \leq k \leq n-3 .
$$

Case 2. $k=n-2$.

Let us consider the first term in $A_{k}$. It contains $I=(22,33, \ldots, n-1 n-1)$, for which

$$
\nabla_{I} \dot{\theta}(0)=\prod_{j=2}^{n-1}\left(2 B+O\left(\Gamma^{1 / 2}\right)+\gamma_{j j}\right)=(2 B)^{n-2}+C(B) O\left(\Gamma^{1 / 2}\right)+C(B) \delta^{3 / 2}
$$

and

$$
\frac{\partial^{n-2} F}{\partial u_{I}}\left(\nabla_{i j} \theta(0)\right)=-\frac{k_{1}}{2} \Gamma+O\left(\Gamma^{3 / 2}\right) .
$$

For different $I$ we have $\left|\nabla_{I} \dot{\theta}(0)\right| \leq C B^{n-3}$ and $\frac{\partial^{n-2} F}{\partial u_{I}}\left(\nabla_{i j} \theta(0)\right)=O(\Gamma)$. 
Therefore the first term in $A_{n-2}$ is equal to

$$
\begin{array}{r}
\lambda^{n-2}\left[(n-2) !\left(-\frac{k_{1}}{2} \Gamma+O\left(\Gamma^{3 / 2}\right)\right)\left((2 B)^{n-2}+C(B) O\left(\Gamma^{1 / 2}\right)+C(B) \delta^{3 / 2}\right)\right. \\
\left.+O\left(B^{n-3}\right) O(\Gamma)\right] \\
=-\frac{k_{1}}{2}(n-2) ! \lambda^{n-2} \Gamma(2 B)^{n-2}\left(1+O\left(\frac{1}{B}\right)+C(B) O\left(\Gamma^{1 / 2}\right)+C(B) \delta^{3 / 2}\right) .
\end{array}
$$

The second term in $A_{n-2}$ is bounded by $C B^{n-4} \Gamma^{2} \lambda^{n-2}$. It follows that

$$
A_{n-2}=-c_{n} \lambda^{n-2} B^{n-2} \Gamma\left(1+O\left(\frac{1}{B}\right)+C(B) O\left(\Gamma^{1 / 2}\right)+C(B) \delta^{3 / 2}\right),
$$

where $c_{n}$ is a strictly positive constant.

To deal with the cases where $k=n-1$ and $n$ we use the following facts.

\section{Lemma II.7.}

$$
\begin{aligned}
& \text { i) } \sum_{|I|=n-1} \frac{\partial^{n-1} F}{\partial u_{I}}\left(\nabla_{i j} \theta(0)\right) \nabla_{I} \dot{\theta}(0)=(n-1) ! \sum_{i, j=1}^{n} \frac{\partial F}{\partial u_{i j}}\left(\nabla_{i j} \dot{\theta}(0)\right) \nabla_{i j} \theta(0) . \\
& \text { ii) } \sum_{|I|=n} \frac{\partial^{n} F}{\partial u_{I}}\left(\nabla_{i j} \theta(0)\right) \nabla_{I} \dot{\theta}(0)=n ! \operatorname{det}\left(\nabla_{i j} \dot{\theta}(0)\right) .
\end{aligned}
$$

Proof. Identify the coefficients of $\lambda^{n-1}$ and $\lambda^{n}$ in the equality

$$
F\left(\nabla_{i j} \theta(0)+\lambda \nabla_{i j} \dot{\theta}(0)\right)=\lambda^{n} F\left(\nabla_{i j} \dot{\theta}(0)+\frac{1}{\lambda} \nabla_{i j} \theta(0)\right) .
$$

Case $k=n-1$. Here we may neglect the terms which are $O\left(\lambda^{n-1} \delta^{3 / 2}\right)$. We consider the first term in $A_{n-1}$ and we use i) of Lemma II.7. By (II.20) we have $\nabla_{i j} \theta(0)=O(\Gamma)$ if $1 \leq i, j \leq n-1$ and $\left|\frac{\partial F}{\partial u_{i j}}\left(\nabla_{i j} \dot{\theta}(0)\right)\right| \leq C(B)$; the corresponding term is then bounded by $\lambda^{n-1} C(B) \Gamma$. We are left with

$$
(1)=2 \sum_{i=1}^{n-1} \frac{\partial F}{\partial u_{i n}}\left(\nabla_{i j} \dot{\theta}(0)\right) \nabla_{i n} \theta(0)+\frac{\partial F}{\partial u_{n n}}\left(\nabla_{i j} \dot{\theta}(0)\right) \nabla_{n n} \theta(0) .
$$

We have $\frac{\partial F}{\partial u_{1 n}}\left(\nabla_{i j} \dot{\theta}(0)\right)=\operatorname{cof}\left(\nabla_{1 n} \dot{\theta}(0)\right)$. If we develop $\operatorname{cof}\left(\nabla_{1 n} \dot{\theta}(0)\right)$ with respect to the first column, all the determinants will be $O\left(\Gamma^{1 / 2}\right)$ because the last line is $O\left(\Gamma^{1 / 2}\right)$, except for the last one, which is equal to

$$
(-1)^{n}\left(b+O\left(\Gamma^{1 / 2}\right)\right)\left((2 B)^{n-2}+C(B) O\left(\Gamma^{1 / 2}\right)\right) .
$$

Therefore

$$
\frac{\partial F}{\partial u_{1 n}}=-b(2 B)^{n-2}+C(B) O\left(\Gamma^{1 / 2}\right)+C(B) \delta^{3 / 2}
$$

and

$$
2 \frac{\partial F}{\partial u_{1 n}}\left(\nabla_{i j} \dot{\theta}(0)\right) \nabla_{1 n} \theta(0)=-b k_{1} x_{1}(2 B)^{n-2}+C(B) O(\Gamma)
$$


For $j=2, \ldots, n-1$ we have $\frac{\partial F}{\partial u_{j n}}\left(\nabla_{i j} \dot{\theta}(0)\right)=b a_{j}(2 B)^{n-3}+C(B) O\left(\Gamma^{1 / 2}\right)$, so

$$
2 \frac{\partial F}{\partial u_{i n}}\left(\nabla_{i j} \dot{\theta}(0)\right) \nabla_{i n} \theta(0)(2 B)^{n-3} b k_{i} a_{i} x_{i}+C(B) O(\Gamma) .
$$

Now $\nabla_{n n} \theta(0)=1$ and $\frac{\partial F}{\partial u_{n n}}\left(\nabla_{i j} \dot{\theta}(0)\right)=\operatorname{cof}\left(\nabla_{n n} \dot{\theta}(0)\right)$. We get

$$
\frac{\partial F}{\partial u_{n n}}=b k_{1} x_{1}(2 B)^{n-2}-\sum_{j=2}^{n-1}\left(a_{j}+\frac{1}{2} b k_{j} x_{j}\right)^{2}(2 B)^{n-3}+C(B) O(\Gamma) .
$$

It follows from (II.31) and (II.32) that

$$
\begin{gathered}
(1)=-b k_{1} x_{1}(2 B)^{n-2}+(2 B)^{n-3} \sum_{j=2}^{n-1} b k_{j} x_{j} a_{j}+b k_{1} x_{1}(2 B)^{n-2} \\
-(2 B)^{n-3} \sum_{j=2}^{n-1}\left(a_{j}+\frac{1}{2} b k_{j} x_{j}\right)^{2}+C(B) O(\Gamma), \\
(1)=-(2 B)^{n-3} \sum_{j=2}^{n-1}\left(a_{j}^{2}+\frac{1}{4} b^{2} k_{j}^{2} x_{j}^{2}\right)+C(B) O(\Gamma) .
\end{gathered}
$$

Let us consider the second term in $A_{n-1}$, which is

$$
\sum_{|I|=n-3} \frac{\partial^{n-2} F}{\partial u_{I} \partial u_{11}}\left(\nabla_{i j} \theta(0)\right) \nabla_{I} \dot{\theta}(0)
$$

it is bounded by $C B^{n-3} \Gamma^{n-(n-2)-1}=C B^{n-3} \Gamma$, by Lemma II.6. Therefore we get

$$
A_{n-1}=-(n-1) ! \lambda^{n-1}(2 B)^{n-3} \sum_{j=2}^{n-1}\left(a_{j}^{2}+\frac{1}{4} b^{2} k_{j}^{2} x_{j}^{2}\right)+\lambda^{n-1} C(B) O(\Gamma) \text {. }
$$

Case $k=n$. By Lemma II.7

$$
\begin{aligned}
A_{n}=\lambda^{n}[\underbrace{n ! \operatorname{det}\left(\nabla_{i j} \dot{\theta}(0)\right)}_{(1)} & \\
& +\underbrace{\left.n(n-1)\left(b^{2}+O\left(\Gamma^{1 / 2}\right)\right) \sum_{|I|=n-2} \frac{\partial^{n-1} F}{\partial u_{I} \partial u_{11}}\left(\nabla_{i j} \theta(0)\right) \nabla_{I} \dot{\theta}(0)\right]}_{(2)} .
\end{aligned}
$$

Let us consider (1). We use (II.27). We develop the determinant with respect to the first column. Then all the determinants are bounded by $C(B) \Gamma^{1 / 2}$, because the last line left is $O\left(\Gamma^{1 / 2}\right)$, except for the last one. For the last determinant we develop with respect to the last column. Then all the determinants will be bounded by $C(B) \Gamma^{1 / 2}$, except for the first one. Then

$$
\begin{aligned}
(1) & =n !(-1)^{n+1} b \cdot(-1)^{n} b(2 B)^{n-2}+C(B) O\left(\Gamma^{1 / 2}\right) \\
& =-n ! b^{2}(2 B)^{n-2}+C(B) O\left(\Gamma^{1 / 2}\right) .
\end{aligned}
$$

Let us consider $(2)$. In $\frac{\partial F}{\partial u_{11}}\left(\nabla_{i j} \theta(0)\right)$ the terms $u_{1 j}$ and $u_{i 1}$ have been cancelled. Let $I$ be a multi-index with $|I|=n-2$. If $I$ contains $(i, j)$ which is different from $(2,2), \ldots,(n-1, n-1)$, then $\nabla_{I} \dot{\theta}(0)$ is $O\left(\Gamma^{1 / 2}\right)$ by (II.27), and the corresponding 
term in $(2)$ is bounded by $C(B) \Gamma^{1 / 2}$. When $I=(22,33, \ldots, n-1 n-1)$ the corresponding term is

$$
\frac{\partial^{n-1} F}{\partial u_{22} \ldots \partial u_{n-1 n-1} \partial u_{11}} \nabla_{22} \dot{\theta}(0) \ldots \nabla_{n-1 n-1} \dot{\theta}(0)=(2 B)^{n-2}+O\left(\Gamma^{1 / 2}\right)
$$

and there are $(n-2)$ ! terms of this form. Therefore

$$
(2)=\left(b^{2}+O\left(\Gamma^{1 / 2}\right)\right) n(n-1)(n-2) !\left[(2 B)^{n-2}+C(B) O\left(\Gamma^{1 / 2}\right)\right] .
$$

It follows from (II.34) and (II.35) that

$$
A_{n}=C(B) O\left(\Gamma^{1 / 2}\right) \lambda^{n} .
$$

Finally, to compute $\operatorname{det}\left(\nabla_{i j} \theta\right)$, me must consider the last term in Lemma 4 , which is

$$
(1)=\sum_{\substack{q=q_{1}+q_{2} \\ q_{1}+2 q_{2}=k \\ q_{2} \geq 1}} \frac{k !}{q_{1} ! q_{2} !} \sum_{\substack{\left|I_{j}\right|=q_{j} \\ I_{2} \neq(11)}} \frac{\partial^{q} F}{\partial u_{I_{1}} \partial u_{I_{2}}}\left(\nabla_{i j} \theta(0)\right) \nabla_{I_{1}} \dot{\theta}(0) \frac{\nabla_{I_{2}} \ddot{\theta}(0)}{2^{q_{2}}} .
$$

Since $q_{2} \geq 1, I_{2} \neq(11)$, we have by (II.18) and (II.19) $\nabla_{I_{2}} \ddot{\theta}(0)=O\left(\Gamma^{1 / 2}\right)$. Then $q=q_{1}+q_{2} \leq k-1$, and Lemma II.6 implies that $\frac{\partial^{q} F}{\partial u_{I_{1}} \partial u_{I_{2}}}\left(\nabla_{i j} \theta(0)\right)=O\left(\Gamma^{n-q-1}\right)=$ $O\left(\Gamma^{n-k}\right)$. We also get, by (II.27), $\left|\nabla_{I_{1}} \dot{\theta}(0)\right| \leq C B$, so

$$
(1)=C(B) O\left(\Gamma^{n-k+\frac{1}{2}}\right) \text {. }
$$

Summing up, it follows from (II.29), (II.30), (II.33), (II.36) and (II.37) that

$$
\begin{gathered}
\operatorname{det}\left(\nabla_{i j} \theta(\lambda)\right) \leq \underbrace{-c_{n} \lambda^{n-2} B^{n-2} \Gamma\left(1+O\left(\frac{1}{B}\right)+C(B) O\left(\Gamma^{1 / 2}\right)+C(B) \delta^{3 / 2}\right)}_{(1)} \\
+\underbrace{\sum_{k=0}^{n-3} \lambda^{k} C_{k} B^{k} \Gamma^{n-k-1}}_{(2)} \underbrace{-\lambda^{n-1}(2 B)^{n-3} \sum_{j=2}^{n-1}\left(a_{j}^{2}+\frac{1}{4} b^{2} k_{j}^{2} x_{j}^{2}\right)}_{(3)} \\
+\underbrace{C(B) \lambda^{n-1} \Gamma}_{(4)}+\underbrace{C(B) \lambda^{n} \Gamma^{1 / 2}}_{(5)}+\underbrace{C(B) \lambda^{n+1}}_{(6)} .
\end{gathered}
$$

Since $\Gamma \leq \delta$ and $\lambda=\frac{\delta}{K}$, the two last terms (5) and (6) are bounded by $C(B) \lambda^{n} \delta^{1 / 2}$. The term (3) is negative. We first choose $B$ so large that $1+O\left(\frac{1}{B}\right) \geq \frac{1}{2}$. Then we write

$$
\text { (4) }=\lambda^{n-2} B^{n-2} \Gamma \cdot \frac{C(B)}{B^{n-2}} \lambda \text {. }
$$


Therefore

$$
\begin{gathered}
\operatorname{det}\left(\nabla_{i j} \theta(\lambda)\right) \leq-c_{n} \lambda^{n-2} B^{n-2} \Gamma\left(\frac{1}{2}+C_{1}(B) O\left(\Gamma^{1 / 2}\right)+C_{2}(B) \lambda+C_{3}(B) \delta^{3 / 2}\right) \\
+\sum_{k=0}^{n-3} C_{k} \lambda^{k} \Gamma^{n-k-1} \cdot B^{n-3}+C(B) \lambda^{n} \delta^{1 / 2} \\
\leq-c_{n} \lambda^{n-2} B^{n-2} \Gamma\left(\frac{1}{2}+C_{1}(B) O\left(\Gamma^{1 / 2}\right)+C_{2}(B) \lambda+\sum_{k=0}^{n-3} C_{k} \Gamma^{n-k-2} \lambda^{k-n+2} \frac{1}{B}\right) \\
+C(B) \lambda^{n} \delta^{1 / 2} .
\end{gathered}
$$

Recall that $\Gamma \leq \delta, \lambda=\frac{\delta}{K}, K \geq 1$, and so

$$
\begin{aligned}
& C_{1}(B) O\left(\Gamma^{1 / 2}\right)+C_{2}(B) \lambda+\frac{1}{B} \sum_{k=0}^{n-3} C_{k} \Gamma^{n-k-2} \lambda^{k-n+2} \\
& \leq C(B) \delta^{1 / 2}+C_{2}(B) \delta+\frac{1}{B} \sum_{k=0}^{n-3} C_{k} K^{n-k-2} .
\end{aligned}
$$

We take $B$ so large that $\frac{1}{B} \sum_{k=0}^{n-3} C_{k} K^{n-2-k} \leq \frac{1}{10}$; then, $B$ being fixed, we take $\delta$ so small that $C(B) \delta^{1 / 2}+C_{2}(B) \delta \leq \frac{1}{10}$.

It follows that

$$
\operatorname{det}\left(\nabla_{i j} \theta(\lambda)\right) \leq-\frac{3}{10} c_{n} \lambda^{n-2} B^{n-2} \Gamma+C(B) \lambda^{n} \delta^{1 / 2} \leq C(B) \lambda^{n} \delta^{1 / 2},
$$

which by (II.15) proves our claim. Now, $\operatorname{det} \nabla_{i j} v(x)=\operatorname{det}\left(\nabla_{i j} u+\nabla_{i j} w\right) \geq$ $\operatorname{det} \nabla_{i j} u$, since $\left(\nabla_{i j} w\right) \geq 0 ;$ so $\operatorname{det} \nabla_{i j} v(x) \geq f(x) \geq c_{0}>C \delta^{1 / 2} \geq \operatorname{det} \nabla_{i j} \rho(x)$ in $V$, which completes the proof of Lemma II.2.

\section{INTERIOR ESTIMATES OF THE SECOND DERIVATIVES}

Let us denote by $\Delta$ the Laplace-Beltrami operator, $\Delta=g^{k \ell} \nabla_{k \ell}$. We consider

$$
w=\log \Delta u+\lambda h,
$$

where $\lambda$ is a positive constant to be chosen and $h$ the function given by (I.1).

Let $p$ be a point at which the continuous function $w$ is maximun on $\bar{\Omega}$. If $p \in \partial \Omega$ then, by the preceeding estimates of the second derivatives on the boundary, we deduce that all the second derivatives are bounded on $\bar{\Omega}$. Therefore we may assume that $p \in \Omega$. At $p$ (which can be taken as the origin) we consider a set of normal coordinates. Recall that then

$$
g_{i j}(p)=\delta_{i j}, \quad \frac{\partial g_{i j}}{\partial x_{k}}(p)=0, \quad \Gamma_{i j}^{k}(p)=0, \quad 1 \leq i, j, k \leq n .
$$

Therefore at $p$ the covariant derivatives agree with the usual one. Without loss of generality we also may assume that at $p$ the matrix $\left(\nabla_{i j} u\right)$ is diagonal, i.e.

$$
\nabla_{i j} u(p)=0 \text { if } i \neq j .
$$

Then at $p$ the matrix $\left(u^{i j}\right)=\left(\nabla_{i j} u\right)^{-1}$ is also diagonal. 
In the sequel we shall use the Einstein sommation convention. Let us set $L=$ $u^{i j} \nabla_{i j}$. It is then easy to see that at $p$

$$
\Delta u L w=\underbrace{L \Delta u}_{(1)}-\underbrace{\frac{u^{i i}(\Delta u)_{i}^{2}}{\Delta u}}_{(2)}+\underbrace{\lambda \Delta u L h}_{(3)} .
$$

First of all, by condition (I.1) we have

$$
\text { (3) } \geq C_{0} \lambda \Delta u \sum_{i=1}^{n} u^{i i}
$$

Next we compute the term (1). If we differentiate the equation $\log \operatorname{det} \nabla_{i j} u=$ $\log f(x)=\Phi(x)$ twice, we get

$$
u^{i j} \nabla_{i j k \ell} u+\nabla_{\ell}\left(u^{i j}\right) \nabla_{i j k} u=\nabla_{k \ell} \Phi
$$

therefore

$$
u^{i j} \nabla_{k \ell i j} u=\nabla_{k \ell} \Phi-\nabla_{\ell}\left(u^{i j}\right) \nabla_{i j k} u+u^{i j}\left(\nabla_{k \ell i j} u-\nabla_{i j k \ell} u\right) .
$$

Multiplying by $g^{k \ell}$ and summing, we get at $p$

$$
L \Delta u=\underbrace{\Delta \Phi}_{(a)}-\underbrace{g^{k k} \nabla_{k}\left(u^{i j}\right) \nabla_{i j k} u}_{(b)}+\underbrace{u^{i i} g^{k k}\left(\nabla_{k k i i} u-\nabla_{i i k k} u\right)}_{(c)} .
$$

By the well known Ricci formulas, $\nabla_{k k i i} u-\nabla_{i i k k} u$ is a linear combination of $\nabla_{p q} u$ and $\nabla_{\ell} u$ with coefficients depending only on the metric $g$ (via the components of the Riemann curvature tensor). Then

$$
|(a)+(c)| \leq C\left(1+\Delta u \sum_{i=1}^{n} u^{i i}+\sum_{i=1}^{n} u^{i i}\right),
$$

where $C$ is under control.

Let us compute the term $(b)$. We have at $p$

$$
\nabla_{k}\left(u^{i j}\right)=-u^{i r} u^{s j} \nabla_{r s k} u=-u^{i i} u^{j j} \nabla_{i j k} u
$$

therefore

$$
(b)=g^{k k} u^{i i} u^{j j}\left(\nabla_{i j k} u\right)^{2} .
$$

Now we use the obvious inequality (for fixed $k$ )

We get using (II.39)

$$
u^{i i} u^{j j}\left(\nabla_{i j k} u-\frac{1}{\Delta u} u_{i k}(\Delta u)_{j}\right)^{2} \geq 0 .
$$

$$
u^{i i} u^{j j}\left(\nabla_{i j k} u\right)^{2} \geq 2 u^{k k} u^{j j} u_{k k} \nabla_{k j k} u \frac{(\Delta u)_{j}}{\Delta u}-\frac{1}{(\Delta u)^{2}} u^{k k} u^{j j} u_{k k}^{2}(\Delta u)_{j}^{2} .
$$

Since $u^{k k} u_{k k}=1$ at $p$, we get

$$
u^{i i} u^{j j}\left(\nabla_{i j k} u\right)^{2} \geq 2 u^{j j} \nabla_{k j k} u \frac{(\Delta u)_{j}}{\Delta u}-u^{j j} u_{k k} \frac{(\Delta u)_{j}^{2}}{(\Delta u)^{2}} .
$$

Multiplying by $g^{k k}$ and taking the sum over $k$, we get

$$
g^{k k} u^{i i} u^{j j}\left(\nabla_{i j k} u\right)^{2} \geq 2 g^{k k} u^{j j} \nabla_{k j k} u \frac{(\Delta u)_{j}}{\Delta u}-u^{j j} \frac{(\Delta u)_{j}^{2}}{\Delta u} .
$$


By the Ricci formulas

$$
g^{k k} \nabla_{k j k} u=g^{k k} \nabla_{k k j} u+R=(\Delta u)_{j}+R,
$$

where $R$ is a linear combination of $u_{\ell}$. Therefore $R$ is under control. It follows that

$$
g^{k k} u^{i i} u^{j j}\left(\nabla_{i j k} u\right)^{2} \geq 2 u^{j j} \frac{(\Delta u)_{j}^{2}}{\Delta u}+2 R u^{j j} \frac{(\Delta u)_{j}}{\Delta u}-u^{j j} \frac{(\Delta u)_{j}^{2}}{\Delta u},
$$

and so

$$
(b) \geq u^{j j} \frac{(\Delta u)_{j}^{2}}{\Delta u}+2 R u^{j j} \frac{(\Delta u)_{j}}{\Delta u} .
$$

By (II.42) to (II.45) we can write

$$
L \Delta u \geq u^{j j} \frac{(\Delta u)_{j}^{2}}{\Delta u}+2 R u^{j j} \frac{(\Delta u)_{j}}{\Delta u}-C_{0}-C_{1} \Delta u \sum_{i=1}^{n} u^{i i}-C_{2} \sum_{i=1}^{n} u^{i i} .
$$

Now since $w$ is maximum at $p$ we have $\nabla_{j} w(p)=0$. Therefore at $p$

$$
\frac{(\Delta u)_{j}}{\Delta u}+\lambda h_{j}=0
$$

It follows that, with a constant $C$ under control, we have

$$
L \Delta u \geq u^{j j} \frac{(\Delta u)_{j}^{2}}{\Delta u}-C \lambda \sum_{j=1}^{n} u^{j j}-C \Delta u \sum_{j=1}^{n} u^{j j}-C \sum_{j=1}^{n} u^{j j}-C .
$$

Coming back to (II.40) and using (II.41), we get, taking $\lambda \geq 1$,

$$
\Delta u \cdot L w \geq-C-C \lambda \sum_{j=1}^{n} u^{j j}-C \Delta u \sum_{j=1}^{n} u^{j j}+C_{0} \lambda \Delta u \sum_{j=1}^{n} u^{j j} .
$$

Now we use the well known inequality

$$
\sum_{j=1}^{n} u^{j j} \geq n\left(\operatorname{det} u^{i j}\right)^{1 / n}=n f^{-1 / n} \geq \alpha_{0} \text { in } \bar{\Omega} .
$$

It follows that

$$
\Delta u \cdot L w \geq-\frac{C}{\alpha_{0}} \sum_{j=1}^{n} u^{j j}-C \lambda \sum_{j=1}^{n} u^{j j}+\left(C_{0} \lambda-C\right) \Delta u \sum_{j=1}^{n} u^{j j} .
$$

We take $\lambda$ so large that $C_{0} \lambda \geq 2 C$, and we use the fact that, since the matrix $\left(u^{i j}\right)$ is positive, we have $L w(p) \leq 0$ at $p$. Therefore

$$
\frac{1}{2} C_{0} \lambda \Delta u(p) \leq C \lambda+\frac{C}{\alpha_{0}},
$$

which shows that $\Delta u(p)$ is under control.

So far we have proved uniform upper bounds for the $C^{2}$ norm on $\bar{\Omega}$ of the solutions. It then follows that the eigenvalues are uniformly bounded below, which implies that the linearized operator of equation (I.2) is actually uniformly elliptic. We may apply the results of Evans [E] (for the interior estimates) and of Krylov $[\mathrm{KR}]$ (see also $[\mathrm{KA}]$ ) (for boundary estimates) to get uniform upper bound for the $C^{2+\alpha}(\bar{\Omega})$ norm. This completes the proof of Theorem I.1. 


\section{REFERENCES}

[A] A. Atallah : Problème de Dirichlet pour des équations de Monge-Ampère réelles relatives à des métriques Riemanniennes, Comm. in P. D. E. 21, (1996), 35-62. MR 96m:35092

[CNS] L. Caffarelli, L. Nirenberg and J. Spruck : The Dirichlet problem for nonlinear second order elliptic equations I, Comm. Pure Appl. Math. 37, (1984), 369-402. MR 87f:35096

[C] C. Corona : Monge-Ampère equations on convex regions of the plane, Comm. in P.D.E., 16 (1), (1991), 43-57. MR 92g:35068

[E] L. Evans : Classical solutions of fully nonlinear convex second order elliptic equations, Comm. Pure Appl. Math. 25, (1982), 333-363. MR 83g:35038

[GS] B. Guan and J. Spruck : Boundary value problems on $S^{n}$ for surfaces of constant Gauss curvature, Annals of Math. 138, (1993), 601-624. MR 94i:53039

[GT] D. Gilbarg and N. Trudinger : Elliptic partial differential equations of second order, 2nd ed., Springer Verlag 1983. MR 86c:35035

[H] J. Hong : Dirichlet problems for general Monge-Ampère equations, Math. Zeitschrift 209, (1992), 289-306. MR 92m:35090

[KA] J. Kazdan : Prescribing the curvature of a Riemannian manifold, CBMS series \# 57, A.M.S. (1985). MR 86h:53001

[KR] N. Krylov : Boundedly inhomogeneous elliptic and parabolic equations in a domain, Math. USSR Izvestia, Vol. 22 n 1, (1984), 67-97. MR 85g:35046

Département de Mathématiques, Université de Paris-Sud, Bât. 425, 91405 Orsay Cedex, France

E-mail address: claude.zuily@math.u-psud.fr 